\title{
The psychiatric case register: noble past, challenging present, but exciting future
}

Gayan Perera, Mishael Soremekun, Gerome Breen and Robert Stewart

\begin{abstract}
Summary
Case registers have been fundamental to mental health research from the early asylum studies onwards. Having declined in popularity over the past 20 years, they are likely to see a resurgence of interest with the advent of electronic clinical records and the technological capacity to derive anonymised databases from these.
\end{abstract}

\section{Declaration of interest}

The authors have been involved in the development and application of a new psychiatric case register at the South London and Maudsley NHS Foundation Trust, and Institute of Psychiatry.
Gayan Perera (pictured) is a South London and Maudsley/King's College London (SLAM/KCL) Biomedical Research Centre PhD student working on the South London and Maudsley Biomedical Research Centre case register. Mishael Soremekun is a research assistant at the $\mathrm{KCL}$ (Institute of Psychiatry) $\mathrm{SLAM} / \mathrm{KCL}$ Biomedical Research Centre Nucleus. Gerome Breen is a postdoctoral research fellow with status of lecturer and SLAM/KCL Biomedical Research Centre Genetics Coordinator. Robert Stewart is a clinical reader, Head of the Section of Epidemiology and joint lead for the SLAM/KCL Biomedical Research Centre Analytic Methods cross-cutting theme.

From the earliest asylum studies onwards, the 'case register' has had a long and influential history in mental health research. Recent moves towards fully electronic clinical records have provided the opportunity for a new generation of case registers supported by technological advances in the capacity to derive anonymised research databases from routine records and to link these with other data repositories. Having been involved in the recent development of one such register, we thought it was timely to review the history and applications of this research resource.

\section{What is a case register?}

Psychiatric case registers have been defined as 'a patient-centred longitudinal record of contacts with a defined set of psychiatric services originating from a defined population.' ${ }^{1}$ The key elements are similar to those for epidemiological research. However, as well as providing important information on the distribution and determinants of relatively rare disorders, case register research can also be used to study outcomes in populations with these disorders. The definition is broad and the level of detail on 'contacts' varies from simple information on demographic status, diagnosis and in-patient service contact through to in-depth freetext data on symptomatology, professional interaction and routinely applied fully structured assessment scales.

\section{History of case registers}

The principle behind case registers is simply the derivation and testing of hypotheses from methodically recorded observations which underpins scientific research. The original motivation to create case registers was influenced by a combination of social, political, professional and technological factors. ${ }^{2}$ Early national case registers began in Norway (from 1936), Denmark and Iceland (incorporating data from 1905 and 1907 respectively) and Israel (1948), followed by several in the USA, Japan (Nagasaki),
Australia (Victoria) and the UK (Camberwell and Aberdeen) in the 1960s, and in Germany (Mannheim), The Netherlands (Groningen) and Italy (Verona) in the 1970s (1973, 1973 and 1978 respectively).

The meetings of a joint inter-register committee from 1980 to 1986 was a high water mark in the UK, representing nine major registers which existed at that time, funded by a variety of sources. However, withdrawal of funding resulted in progressive and complete closure from 1985 to 1987. It is beyond the scope of this article to discuss in detail the reasons for this decline although they include a loss of academic interest and decreasing integration with clinical services, rendering the careful recording of researchrelevant data less acceptable to clinical staff. Case registers have continued internationally including long-running programmes in Groningen, Verona and Victoria and national registers in Israel, Denmark and New Zealand.

\section{Uses of case registers}

Case registers contribute important data for service planning, epidemiological, administrative and operational research. ${ }^{1}$ The research contribution is clearly determined by the format of the register and type of data collected. Case registers have been used to investigate specific questions about health service delivery and to evaluate care programmes and patterns of care, as well as the validity of diagnostic tools and therapies. In epidemiological research, they have particular advantages for describing morbidity and service use associated with severe mental disorders because of the problems of inadequate screening instruments for severe disorders and of selective non-participation in community surveys. Well-established case registers with informative individual data are ideal for cohort studies, providing the principal means to investigate factors predicting course and outcome in people with a defined disorder as well as disorder incidence (particularly useful for relatively rare and severe disorders) and predictors of this. ${ }^{3}$ Within outcomes research, case registers are particularly important for evaluating costs of mental healthcare ${ }^{4}$ and, through data linkage, mortality. ${ }^{5}$ Case register-based cohort studies are most often historical in design (with outcome already ascertained at the point of analysis commencement) and therefore offer cost and logistical advantages. Some registers have been developed to incorporate computer programs which can select cohorts from the main body of register participants. Other uses of registers include facilitating the recruitment of potential cases for casecontrol investigations of potential risk factors. 
Table 1 Description of papers indicating use of a psychiatric case register derived from a literature review carried out for publications in five 2 -year periods over 40 years

\begin{tabular}{|c|c|c|c|c|c|c|}
\hline Study design & $1964-65$ & $1974-75$ & $1984-85$ & 1994-95 & 2004-05 & Total \\
\hline Case-control design within the register & & 1 & 1 & 3 & 7 & 12 \\
\hline Cohort study specifically for mortality & & 3 & 1 & 4 & 4 & 12 \\
\hline Cohort study with external comparison group & & 2 & 1 & 5 & 11 & 19 \\
\hline Cohort study within the register & 1 & 3 & 5 & 14 & 24 & 47 \\
\hline Cross-sectional descriptive study & 1 & 6 & 11 & 14 & 9 & 41 \\
\hline Observational intervention study (after and before reform) & & & 3 & 1 & 5 & 9 \\
\hline Provision of cases with external controls & & & & 2 & 5 & 7 \\
\hline Provision of participants for intervention study & & & & 1 & 3 & 4 \\
\hline Total & 2 & 15 & 22 & 44 & 68 & 151 \\
\hline
\end{tabular}

In reviewing the application of case registers, we carried out a PubMed and Google Scholar literature search for the MeSH term 'psychiatric case registers' screening 2-year periods over the past five decades (2004-05, 1994-95, 1984-85, 1974-75, 1964-65). The type of study design and objectives for the 151 articles identified are summarised in Table 1. Over the 40-year period there was a clearly marked increase in publications citing the use of a case register, although this publication count remained low considering the volume of mental health research. In general, over the same period, the profile of study design has also changed with a decline in simple cross-sectional descriptive analyses, and an increase in cohort studies and the use of registers to provide cases for case-control studies.

\section{Strengths and limitations}

The principal strengths of case registers are their size and prospective design, as well as the specific data (e.g. on course, outcome, service use and cost) which tend not to be collected elsewhere. Whether used for cohort studies or to generate cases for case-control designs, case registers are most valuable for research into disorders that are relatively rare (i.e. will not appear with sufficient frequency in community studies) and with a high chance of secondary care contact (i.e. where people with the disorder who are known to secondary care services are reasonably representative of total cases in the source community). In mental health these principles apply most strongly to psychotic disorders and least strongly to common mental disorders.

One limitation of case registers lies in the fact that the information they contain is less likely to have been recorded in a standardised way and is more inaccurate than that obtained in a dedicated research project. Goodman ${ }^{6}$ noted that the diagnosis recorded was particularly unreliable. However, a World Health Organization collaborative study in the UK found a relatively high (85\%) concordance between a psychiatric case register and ICD-9 diagnosis. ${ }^{7}$ Maintaining accuracy (i.e. keeping records up to date) is also a challenge.

A second limitation (inherent to observational research) is that causal inferences are limited for observed associations, principally because residual confounding can never be effectively excluded as it can in a randomised controlled trial. However, the advantage of a case register cohort study over a trial is that the scale can be many magnitudes larger. For example, a single centre's case register may well have data on a larger number of people with a particular disorder receiving different interventions, and longer follow-up, than all international trials combined. This is particularly important for rare outcomes such as medication side-effects or secondary benefits not originally envisaged, which trials are not powered to detect. Compared with most trials, case registers also offer the advantages of a naturalistic setting and therefore a broader and more generalisable evidence base.

A particular challenge for case registers concerns the confidentiality and privacy of the information. Legal frameworks for this vary between nations. In Norway, for example, a psychiatric case register founded in 1916, covering a population of 8 million, was abolished in 1988 because of public concerns about confidentiality. ${ }^{8}$ A broader issue concerns stakeholder participation and the need for case registers, where operating, to address the questions most important to users and policy makers rather than necessarily those of academics. A review of recent case register output was critical in this respect and concluded that important questions were not being addressed. ${ }^{9}$

\section{Looking to the future}

There remains a need for internationally comparable morbidity statistics (e.g. outlined in the European Commission's recent DG SANCO health plan; http://www.epha.org/a/3051). Early case registers relied on considerable goodwill and close working relationships between academic and clinical colleagues. However, the major drawback was always that the data had to be manually recorded and entered into computers, as these became available, for analysis. This placed substantial limits on the depth of information that could be contained and, therefore, on the research output that could be generated. Several relatively recent developments suggest that there may be substantial future possibilities for this study design. First, with current trends towards paperless clinical services, the volume of potential information already in electronic format is vast. Second, in a UK context at least, increasing requirements for service providers to generate activity data for funding has meant that clinical records are increasingly structured and available for analysis. Third, technological advances now make it possible for both free-text and fixed-variable fields to be searched and extracted into anonymised databases for analysis (the requirements for the recently developed South London and Maudsley case register). Fourth, similar developments are occurring in a variety of health service settings with, in the UK, a national organisation currently set up to consider the highly complex technical, ethical and security issues surrounding database linkage (Connecting for Health NHS Research Capability Programme; http://www. connectingforhealth.nhs.uk/systemsandservices/research). Fifth, if not already capable, current technology is at the cusp of being able to handle the very large information resources generated when 
biological and imaging databases from nested research projects are also incorporated: possibly the development that is most likely to shape the case registers of the future.

Gayan Perera, MSC, Mishael Soremekun, MSc, Gerome Breen, PhD, Robert Stewart, MD, MRCPsych, King's College London (Institute of Psychiatry), UK

Correspondence: Robert Stewart, Section of Epidemiology (Box 60), Institute of Psychiatry, King's College London, De Crespigny Park, London SE5 8AF, UK. Email: r.stewart@iop.kcl.ac.uk

First received 13 May 2009, final revision 13 May 2009, accepted 9 Jun 2009

\section{Funding}

All authors are funded by the NIHR Biomedical Research Centre for Mental Health, the South London and Maudsley NHS Foundation Trust, and the Institute of Psychiatry, King's college London.

\section{References}

1 Ten Horn GHMM. A classification of different types of psychiatric case registers. In Psychiatric Case Registers in Public Health (ed GHMM Ten Horn R Giel, WH Gulbinat, Henderson JH): 388-401. Elsevier, 1986.
2 Fryers T, Greatorex I. Case registers and mental health information systems. In Measuring Mental Health Needs (eds G Thomicroft, CR Brewin, J Wing): 81-98. Gaskell, 1992.

3 Häfner $\mathrm{H}$, Maurer $\mathrm{K}$, Löffler W, an der Heiden W, Munk-Jørgensen P, Hambrecht $M$, et al. The ABC Schizophrenia Study: a preliminary overview of the results. Soc Psychiatry Psychiatr Epidemiol 1998; 33: 380-6.

4 Amaddeo F, Beecham J, Bonizzato $\mathrm{P}$, Fenyo A, Tansella M, Knapp M. The costs of community-based psychiatric care for first-ever patients. A case register study. Psychol Med 1998; 28: 173-83.

5 Hiroeh U, Appleby L, Mortensen PB, Dunn G. Death by homicide, suicide, and other unnatural causes in people with mental illness: a population-based study. Lancet 2001; 358: 2110-2.

6 Goodman $A B$, Rahav M, Popper M, Ginath $Y$, Pearl E. The reliability of psychiatric diagnosis in Israel's Psychiatric Case Register. Acta Psychiatr Scand 1984; 69: 391-7.

7 Jones SJ, Cooper JE, Davis N. Diagnostic validity and comparability. In Psychiatric Case Registers in Public Health. A Worldwide Inventory (eds GHMM Ten Horn, R Giel, H Gulbinat, JH Henderson): 175-8. Elsevier، 1986.

8 Munk-Jorgensen $\mathrm{P}$, Kastrup M, Mortensen PB. The Danish Psychiatric register as a tool in epidemiology. Acta Psychiatrica Scandinavica 1993; 82: 130-5.

9 Wierdsma Al, Sytema S, van Os JJ, Mulder CL. Case registers in psychiatry: do they still have a role for research and service monitoring? Curr Opin Psychiatry 2008; 21: 379-84. 